\title{
Flexible Gripper, Design and Control for Soft Robotics
}

\author{
Catalina Castillo-Rodriguez ${ }^{1}$, Robinson Jimenez-Moreno ${ }^{2}$. \\ ${ }^{1,2}$ Department of Mechatronics Engineering, Universidad Militar Nueva Granada, Colombia
}

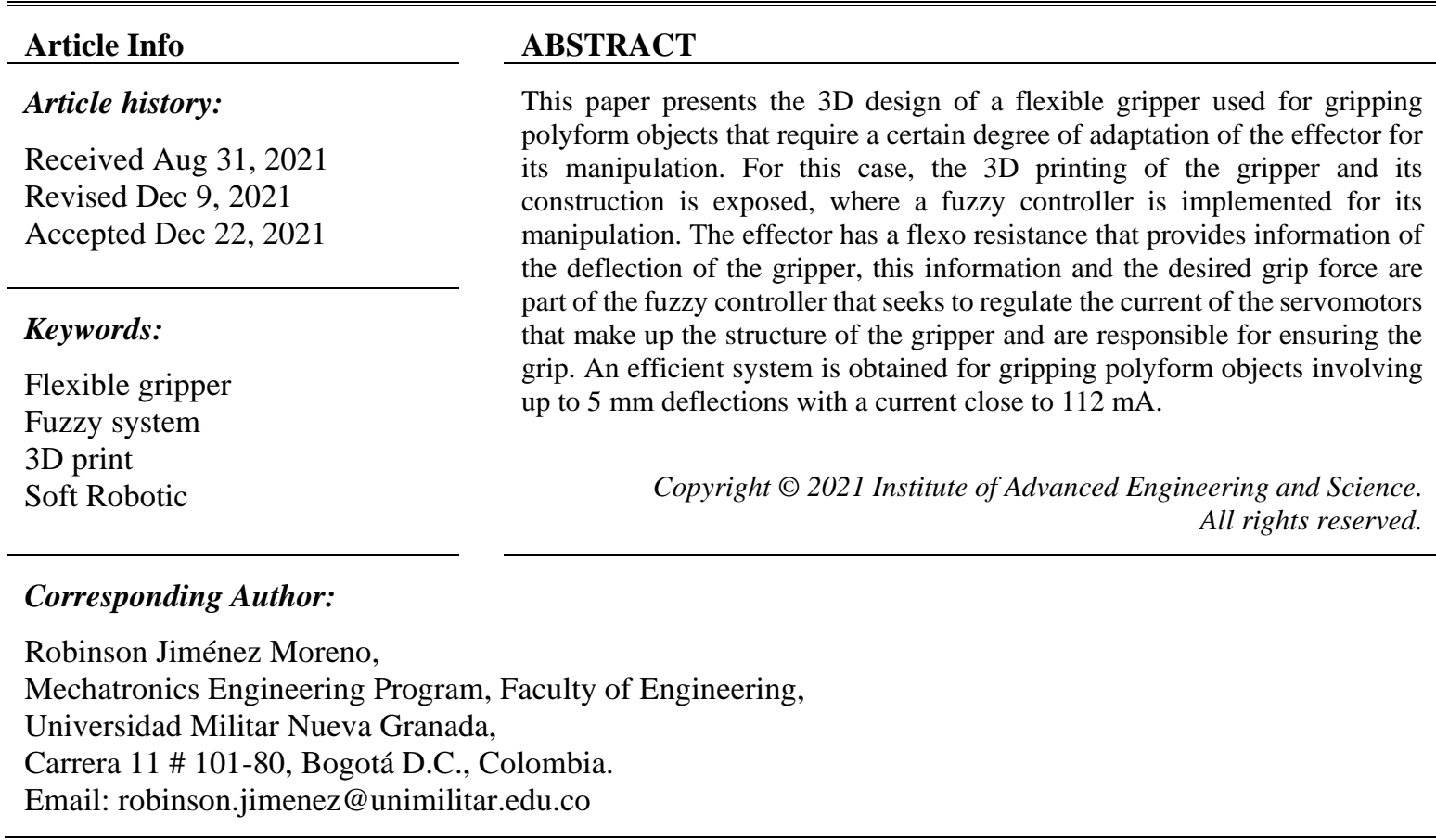

\section{INTRODUCTION}

In the field of robotics, many new technologies and materials have been introduced to improve robot functionality; in recent years, these include 3-D printing as an opportunity to fabricate novel structures, as mentioned in [1]. The development of robotic structures as actuators is of great interest, as evidenced by the work developed in [2][3] and [4], to name a few. The 3-D printing systems allow the development of various types of actuators with flexible structures of different types of material, renewing the conventional robots as mentioned in [5].

Modern robotic systems are starting to employ flexible grippers oriented to soft robotics, as discussed in [6], where one of the antecedents for this type of design is discussed in [7]. In [8] and [9], the use of 3D printing in the design of flexible grippers for complex, gripping objects with robots is illustrated, both are based on a three-finger design, but in extremes of flexibility, the first very flexible and the second with a lesser degree.

This article exposes a midpoint of rigidity in the design of a flexible gripper by 3D printing, obtaining a base structure like the one exposed in [9] but having greater dimension and internal separation area per finger allows greater flexibility. Other flexible gripper models can be found in [10], [11], and [12].

Factors such as versatility, functionality and efficiency are crucial when establishing the devices implemented within an assembly line [14]. Therefore, elements such as grippers are widely required in processes such as pick and place, where a manipulator is required to have the ability to interact with different parts within its environment employing a gripper [14]. The flexible effector concept can adapt to the shape of the object to obtain an optimal grip [15]. It is for this reason that Festo, world leader in automation and robotics, offers its customers different models of adaptive effectors whose methodology is based on biological models exposed throughout nature [16] and that are the basis for the continuity of work in articles such as those mentioned above, the one exposed in [17] and others.

The flexible effector shown is developed as part of an assistive robot capable of interacting with a user in residential environments, i.e., where the objects to be grasped by the robot require the effector to be able to adopt shapes close to the grasping objects. This article is divided into four sections, the present 
introduction, section two, which describes the methodology employed, section three which presents the analysis of results and the final section which presents the conclusions reached.

\section{RESEARCH METHOD}

Figure 1 exposes the system planning diagram, which is established in two stages of mechanical and electronic design, to develop a flexible robotic effector manufactured by $3 \mathrm{D}$ printing. The system is controlled by an algorithm that allows the movement of 2 servomotors by deforming a resistive bending sensor located on one of the gripper's gripper faces. The system is mechatronic, so it involves 3 stages: design, manufacturing and control algorithm, which are discussed below.

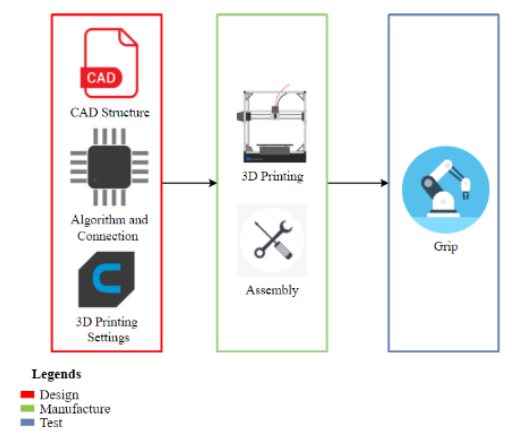

Figure 1. System Planning Diagram.

\subsection{Design}

For the design we determine the components to be used to manufacture the device, based on the mechanical structure of the system by means of additive manufacturing implementing a PLA filament (polylactic acid), which is one of the most used materials for printing mechanical prototypes in research developments, in turn the effector will be assembled by means of commercial screws.

The manipulation of the gripper structure is assigned to the electronic part of the system. An Arduino microcontroller is selected, which is the basis for the development of the algorithm that controls two metal pinion gear servomotors with 360 degrees rotation capacity. This process depends on the degree of angular deformation of a resistive bending sensor. The references of the implemented components are listed in Table 1 .

Table 1. Components of the System

\begin{tabular}{cccc}
\hline & Component & Units & Reference \\
\hline \multirow{2}{*}{ Mechanic } & 3D printing filament & 9 & PLA Hatchbox \\
& Screw & 6 & M5 x 15 mm \\
Electronic & Servomotor & 2 & MG90S Tower Pro \\
& Flexo Sensor & 1 & SEN-10264 Spectralsymbol \\
& Microcontroller & 1 & Arduino Mega \\
\hline
\end{tabular}

The mechanical design of the robotic effector, is inspired by the flexible mechanism exposed by Festo of the MultiChoice Gripper [16], where the grippers play a main role because they flex in order to adapt to the shape of the gripping object. The system consists of 5 CAD parts shown in Figure 2, whose files were downloaded from an open source platform in order to perform printing tests by varying their configuration. In this case it is a gripper type gripper with only opening and closing movements, without rotation.

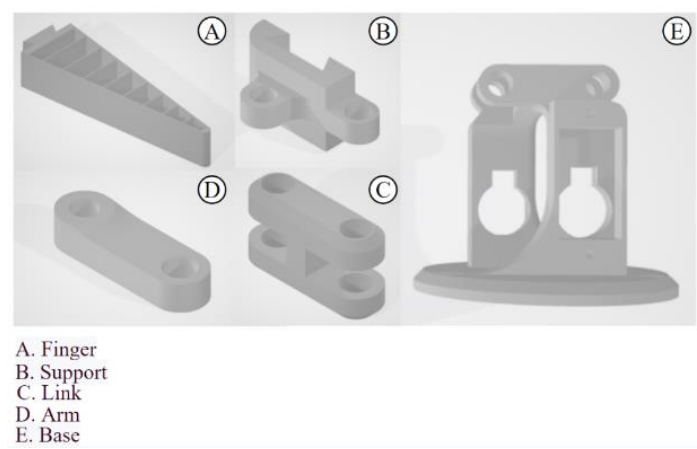

Figure 2. CAD parts of the Robotic Effector. 


\subsection{Manufacturing}

The printed structure is illustrated in Figure 3, where the finish of each piece (A-E) is evident after a total printing period of 3 hours and 41 minutes. As mentioned, the prototype was printed using PLA, a material known for its resistance to deformation, it is for this reason that the design and configuration of the printing of the grippers (A) must consider the direction of the forces at the time of grip.

A rotational to linear reciprocating motion transmission mechanism known as crank-crank is implemented, where the parts $(\mathrm{B}, \mathrm{C})$ play as the crank, while the arm (D) would be the connecting rod because the servomotors were embedded in the base $(\mathrm{E})$ and the shafts generate a rotational motion which the arm will transmit. Figure 4 illustrates the assembled sensor.

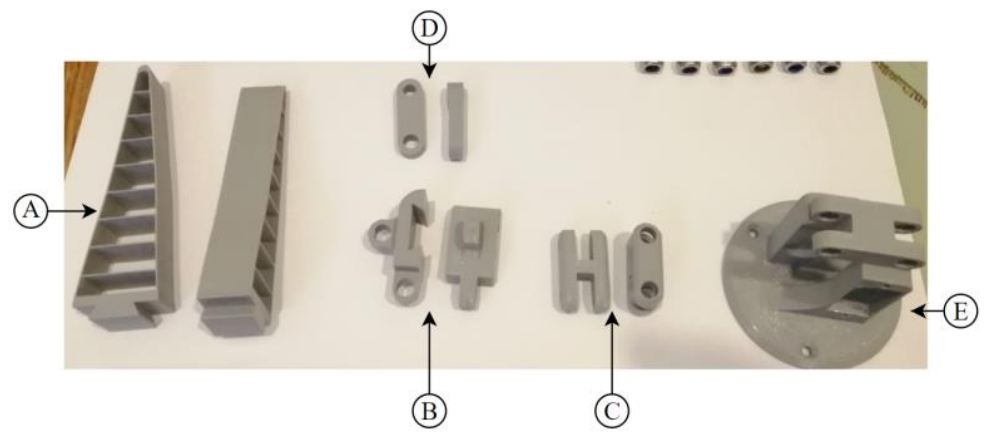

Figure 3. 3D Printed Structure of the Robotic Effector.

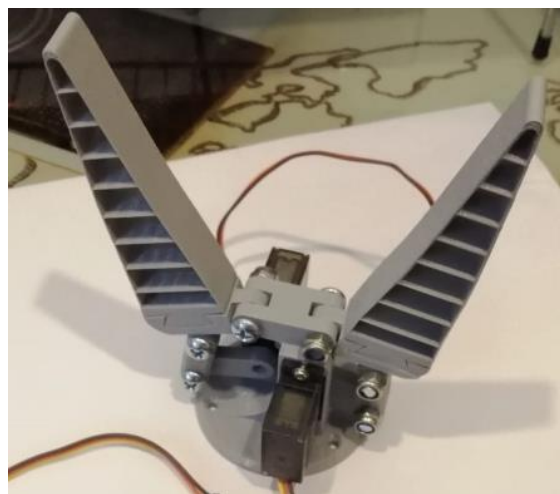

Figure 4. 3D structure of the assembled Robotic Effector.

\subsection{Control algorithm}

The electronic system is composed of an Arduino Mega 2560 reference microcontroller, two MG90S servomotors, a 150k resistor and a SEN-10264 flexo sensor, this sensor is attached to one of the lateral segments of the gripper to measure the deformation. Fig. 5 shows the connection diagram of the system implemented to develop the grip control. The system has as physical input the flexo sensor and outputs the servomotors. The PLA used has a standard mechanical deflection resistance of $485 \mathrm{Kg} / \mathrm{cm}^{2}$, which is above the torque that can be applied by the motor of $2 \mathrm{Kg} / \mathrm{cm}$.

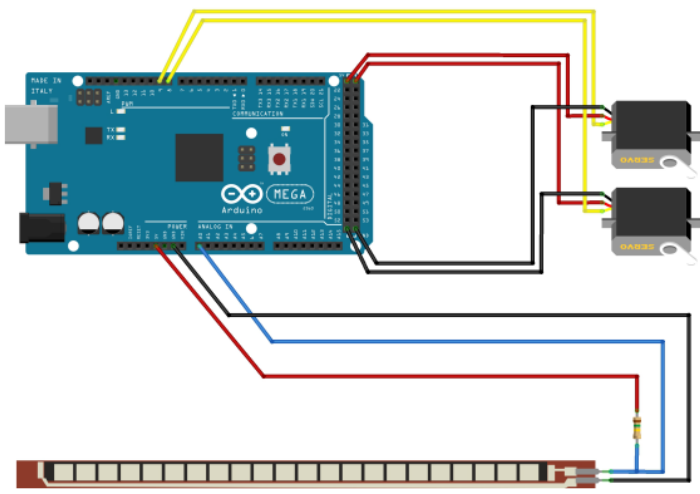

Figure 5. System Connection Diagram. 
In principle, the resistive bending sensor is a potentiometer that varies depending on the degree of inclination of the same. This is composed of a plastic strip with a conductive coating, when it is straight it has a nominal value of resistance $(25 \mathrm{~K})$ but when this is bent increases the value of the resistance to double the nominal value, this mechanism is illustrated in Fig 6 . The selected reference has a tolerance of $+/-30 \%$.

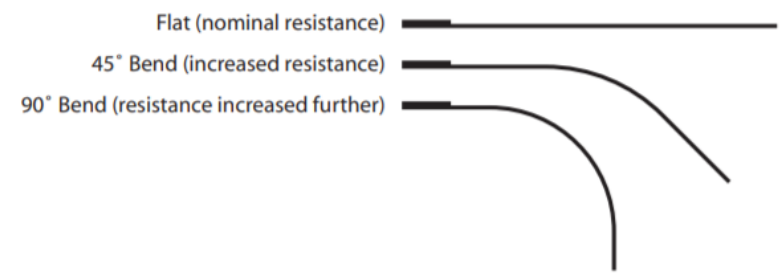

Figure 6. Deformation of the Spectralsymbol Flexo Sensor.

The algorithm for the control of the servomotors is described by the flowchart illustrated in Figure 7, first initialize the analog input to pin A0 for flexo resistance and digital outputs with pins D9 and D8 for each servomotor respectively. Then due to the voltage division circuit an integer value between $0-1023$ is returned corresponding to the voltage value between $0-5$ volts at the time of varying the resistance. The input and output processing algorithm is performed using a fuzzy inference system described in the next section.

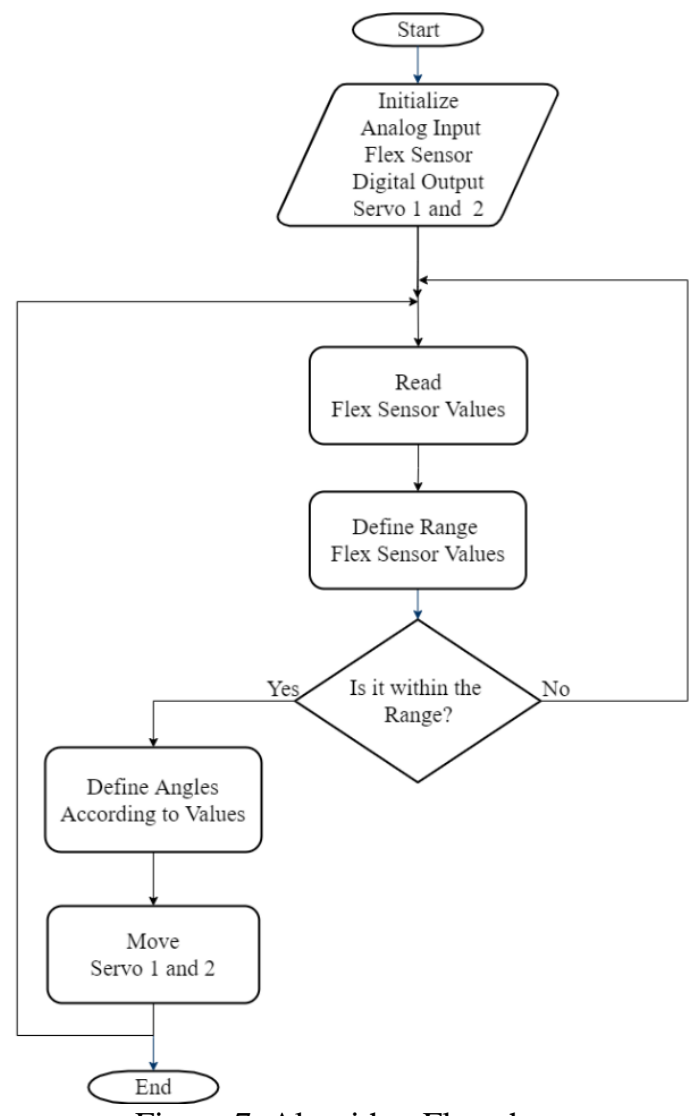

Figure 7. Algorithm Flowchart.

\section{RESULTS AND DISCUSSION}

Given the non-linearity of the system due to the different deformations that the effector can take, depending on the object to be grabbed, the classical control techniques are not the most recommended strategy for this case, as illustrated in the state of the art, in the introduction section, the fuzzy systems present an efficient alternative for its manipulation.

To control the gripper, the fuzzy control system is established (see Fig 8), which operates depending on the desired gripper force, which is according to the type of object to be gripped. The aim is to control the operating current of the servomotor to secure the grip, which is dependent on the deformation of the gripper. 
Table 2 illustrates the control rules and Figure 9 illustrates the respective membership functions, where the universe of discourse for the grip goes from 0 to $100 \%$, the universe of discourse for the deformation (D) goes from 0 to 5 millimeters and the universe of discourse for the current goes from 0 to 120 milliamperes, according to the specifications of the servomotor.

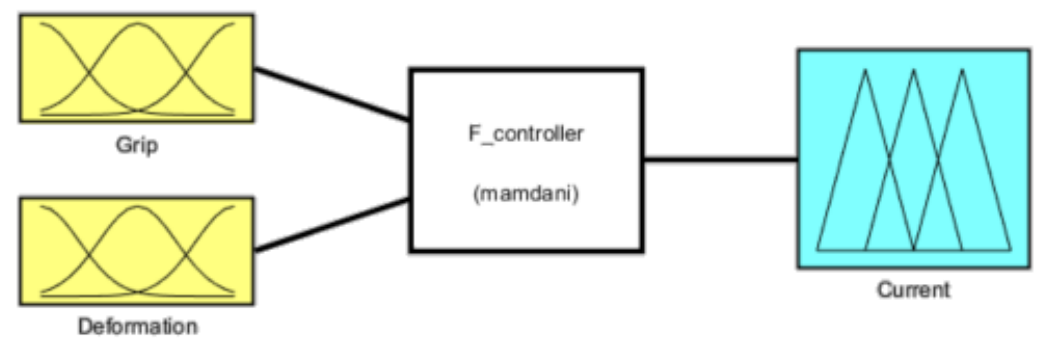

Figure 8. Fuzzy inference system.

Table 2. Fuzzy System Rule Base

\begin{tabular}{cccc}
\hline GRIP $\backslash$ DEFORMATION & LD & MD & HD \\
\hline SOFT & MLC & MLC & MC \\
MEDIUM & MLC & MC & MHC \\
STRONG & MC & MHC & MHC \\
\hline
\end{tabular}

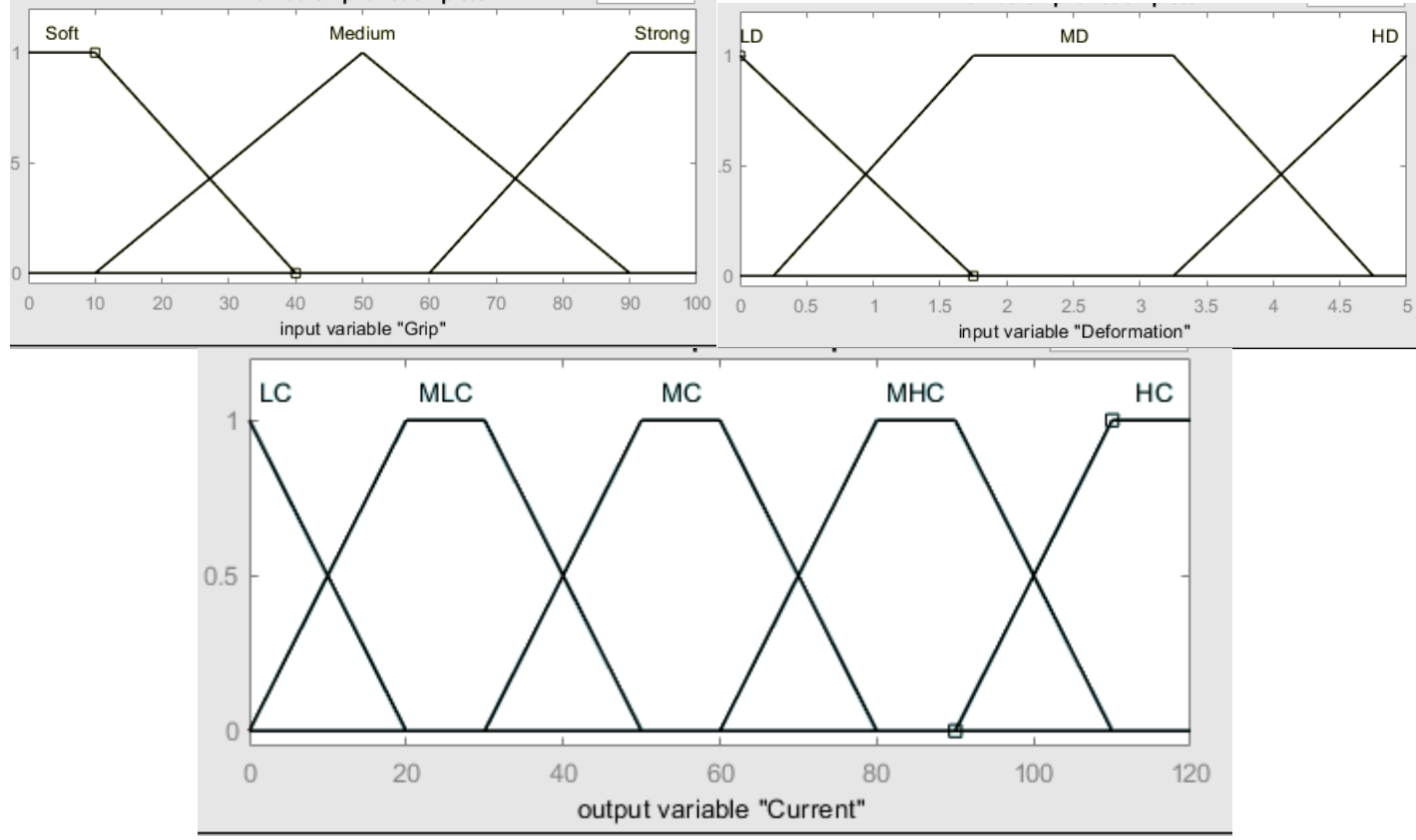

Figure 9. Input and output fuzzy sets.

Fig 10 shows the result of the control algorithm for two extreme cases, the left part presents a soft grip of a plastic cup, while the right part presents a strong grip with maximum deformation of the gripper for a grip of a glass container. It is evident that for a strong grip the current increases significantly compared to the soft grip, as shown in Table 3. The force required to deform the gripper and firmly hold glass is given by the current that increases the torque of the servomotors, forcing the movement that is reflected in the deformation, the sensor feedback allows the control strategy. The deformation is due to the contour of the object to be gripped, once it is released, the effector returns to its initial shape. 

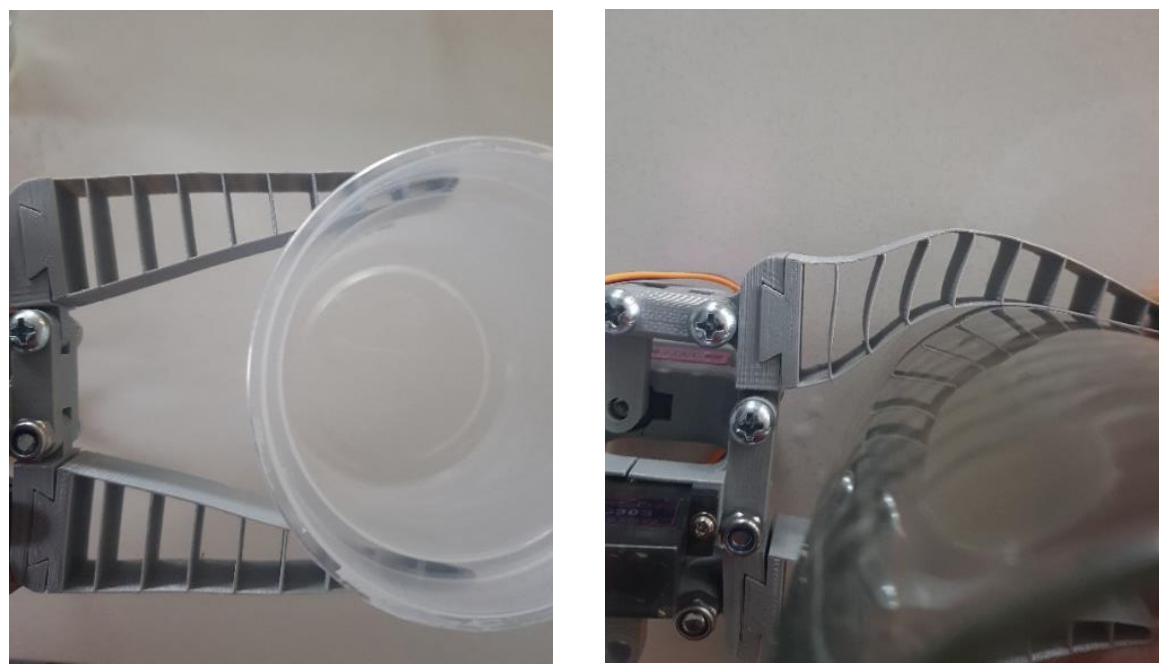

Figure 10. Deformation for gripping rigid objects.

\begin{tabular}{ccc}
\multicolumn{3}{c}{ Tabla 3. Control input/output ratio } \\
\hline GRIP & $\begin{array}{c}\text { DEFORMATION } \\
(\mathrm{mm})\end{array}$ & Current (mA) \\
\hline SOFT & 1 & 18 \\
MEDIUM & 3 & 79 \\
STRONG & 5 & 112 \\
\hline
\end{tabular}

\section{CONCLUSIONS}

It is evidenced the little deformation of the internal fins of the grippers, for this reason it is proposed to create a rotation mechanism in the supports in order to obtain a bending without permanent deformation of the lateral layer of the grippers. It is also proposed to make a rubber covering to improve the grip of objects with smooth surfaces.

With regard to the printing of the 3D model of the gripper, it is evident that the advanced generation of supports using the interface proposed by Cura, showed an improvement compared to the supports generated with Meshmixer, due to the ease with which they were removed from the base piece.

The implementation of the gripping system for objects of different stiffness is one of the strong applications of the flexible grippers based systems, as it was evidenced, requiring a torque control through current to achieve grips of different shape, for which the implemented fuzzy controller demonstrated its efficiency in this task.

\section{ACKNOWLEDGMENTS}

The authors are grateful to the Militar Nueva Granada University, which through its Vice chancellor for research finances the present project with code PIC-ING-3382 and titled "Controlador de gripper flexible", from which the present work is derived.

\section{REFERENCES}

[1] P. J. French, G. J. Krijnen, S. Vollebregt and M. Mastrangeli, "Technology Development for MEMS: A Tutorial," in IEEE Sensors Journal, doi: 10.1109/JSEN.2021.3104715.

[2] B. Gorissen, T. Chishiro, S. Shimomura, M. De Volder, D. Reynaerts and S. Konishi, "Flexible pneumatic twisting actuators," 2013 Transducers \& Eurosensors XXVII: The 17th International Conference on Solid-State Sensors, Actuators and Microsystems (TRANSDUCERS \& EUROSENSORS XXVII), 2013, pp. 1687-1690, doi: 10.1109/Transducers.2013.6627110.

[3] M. Komagata, Y. Imashiro, K. Yamamoto and Y. Nakamura, "Preferred Oil and Ceramics Options for EHA Drive Systems and Computed Torque Control of an EHA-Driven Robot Manipulator," 2021 30th IEEE International Conference on Robot \& Human Interactive Communication (RO-MAN), 2021, pp. 540-545, doi: 10.1109/ROMAN50785.2021.9515398.

[4] Y. Do, D. -S. Choi, G. Shimoga and S. -Y. Kim, "Wavy Silicone Rubber Based Flexible Vibrotactile Actuator," 2019 19th International Conference on Control, Automation and Systems (ICCAS), 2019, pp. 1382-1384, doi: 10.23919/ICCAS47443.2019.8971491.

[5] Y. Yamada and T. Nakamura, "Actuatable Flexible Large Structure Using a Laminated Foam-based Soft Actuator," 2020 IEEE/SICE International Symposium on System Integration (SII), 2020, pp. 74-79, doi: 10.1109/SII46433.2020.9026003. 
[6] J. Guo, C. Xiang, P. Zanini and J. Rossiter, "Magnetic Augmented Self-sensing Flexible Electroadhesive Grippers," in IEEE Robotics and Automation Letters, vol. 4, no. 3, pp. 2364-2369, July 2019, doi: 10.1109/LRA.2019.2903570.

[7] Y. Bi-qiang, W. Hai-shan and Q. Li-fang, "Design and optimization of micro flexible gripper," 2011 International Conference on Consumer Electronics, Communications and Networks (CECNet), 2011, pp. 4890-4893, doi: 10.1109/CECNET.2011.5769024.

[8] R. Sadeghian, P. Sedigh, P. Azizinezhad, S. Shahin and M. T. Masouleh, "Design, Development and Control of a Three Flexible-Fingers Gripper Based On Hand Gesture," 2018 6th RSI International Conference on Robotics and Mechatronics (IcRoM), 2018, pp. 359-363, doi: 10.1109/ICRoM.2018.8657517.

[9] A. TONG, J. -y. HU, M. -h. ZHANG, Y. JIN and J. -k. DU, "Flexible Gripper Manufacturing and Simulation Based on 3D Printing," 2019 13th Symposium on Piezoelectrcity, Acoustic Waves and Device Applications (SPAWDA), 2019, pp. 1-4, doi: 10.1109/SPAWDA.2019.8681858.

[10] C. Chen and W. Chong, "Force controlled robot gripper with flexible joint for delicate assembly task," 2013 13th International Conference on Control, Automation and Systems (ICCAS 2013), 2013, pp. 935-939, doi: 10.1109/ICCAS.2013.6704049.

[11] W. Mann, G. Peschl and C. Wogerer, "Development of flexible gripper for precision assembly of cylinder locks," 2007 IEEE International Symposium on Assembly and Manufacturing, 2007, pp. 19-24, doi: 10.1109/ISAM.2007.4288443. L. Te, L. Tian, L. Heng, C. Boyao, L. Zhenkun and W. Yongqing, "Bionic Design and Casting Forming Method of a Soft Gripper Robot," 2020 3rd International Conference on Mechatronics, Robotics and Automation (ICMRA), 2020, pp. 116-120, doi: 10.1109/ICMRA51221.2020.9398370.

[12] C.I. Basson, G. Bright y A.J. Walker. "Testing flexible grippers for geometric and surface grasping conformity in reconfigurable assembly systems.” En: South African Journal of Industrial Engineering 29.1 (2018), págs. 128-142. ISSN: 2224-7890.

[13] Anand Mohan, Gokul Soman, Srichitra S y Jaimon Cletus. "Design and Simulation of 3 Fingered Underactuated Gripper”. En: 2020 IEEE Recent Advances in Intelligent Computational Systems (RAICS). 2020, págs. 86-90. DOI:10.1109/RAICS51191.2020.9332490.

[14] Vaidehi Patil, Tanisha Narnaware, Narendra Gangwani y N.R. Raykar. "Development of flexible universal gripper for handling light weight parts of arbitrary shape.” En: Materials Today: Proceedings 28.Part 4 (2020), págs. 2591 2598. ISSN: 2214-7853.

[15] An TONG, Jian-ying HU, Ming-hua ZHANG, Yuan JIN y Jian-ke DU. "Flexible Gripper Manufacturing and Simulation Based on 3D Printing”. En: 2019 13th Symposium on Piezoelectrcity, Acoustic Waves and Device Applications (SPAWDA). 2019, págs. 1-4. DOI: 10.1109/ SPAWDA.2019.8681858.

[16] Festo AG \& Co.KG. "MultiChoiceGripper". En: Variable gripping based on human hand (2018).

[17] Mengying Xie, Mingzhu Zhu, Zhaoshu Yang, Shima Okada y Sadao Kawamura. "Flexible self-powered multifunctionalsensor for stiffness-tunable soft robotic gripper by multimaterial 3D printing." En: 79 (2021).

\section{BIOGRAPHY OF AUTHORS}

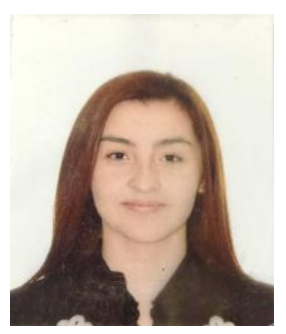

Catalina Castillo-Rodriguez is a mechatronic engineering student at MilitarNueva Granada University, Bogota, Colombia. Currently she is a research assistant with DAVINCI research group, where the main lines of investigation are robotics and artificial intelligence. Some her works are the scientific initiation projects titles "3D printed Flexible End Effector" and "Flexible Gripper Controller".

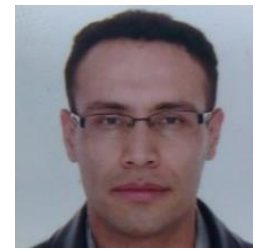

Robinson Jiménez-Moreno is an Electronic Engineer graduated from the Francisco José de Caldas District University in 2002. He received a M.Sc. in Engineering from the National University of Colombia in 2012 and Ph.D in Engineering at the Francisco José de Caldas District University in 2018. His current working as assistant professor of Universidad Militar Nueva Granada and research focuses on the use of convolutional neural networks for object recognition and image processing for robotic applications such as human-machine interaction. 